\title{
Efeito de anos e determinação do coeficiente de repetibilidade de características de frutos de goiabeira-serrana ${ }^{(1)}$
}

\author{
Juliana Degenhardt(2), Jean Pierre Ducroquet ${ }^{(3)}$, Mauricio Sedrez dos Reis ${ }^{(2)}$, \\ Miguel Pedro Guerra(2) e Rubens Onofre Nodari(2)
}

\begin{abstract}
Resumo - A variância ambiental costuma ser bastante significativa em características de produção e de frutos, a maioria de herança quantitativa. Este estudo teve por objetivo avaliar o efeito de anos e determinar o número de anos necessários para avaliação precisa do peso do fruto, peso de casca, comprimento, diâmetro, relação comprimento/diâmetro, rendimento de polpa e sólidos solúveis totais dos frutos de goiabeira-serrana (Acca sellowiana), em três anos de avaliação. Com exceção de sólidos solúveis totais, o efeito de anos representou grande parte da variância total. A partir do coeficiente de repetibilidade, determinou-se que seriam necessários no mínimo de quatro a seis anos de avaliação para seleção das plantas, com precisão de $80 \%$.
\end{abstract}

Termos para indexação: Acca sellowiana, herdabilidade, fator ambiental, variação genética.

\section{Effects of years and determination of repeatability of some characteristics of feijoa fruits}

\begin{abstract}
The environmental variance is quite significant in production and fruits traits, most of quantitative inheritance nature. This study evaluated the effect of years and the determination of the minimum number of necessary years for evaluation one needs for fruit weight, peel weight, length, diameter, relationship length/diameter, pulp revenue and soluble solids contents of fruits of feijoa (Acca sellowiana). Except for soluble solids contents, the effect of years represented great part of the total variance. Based on the repeatability coefficient, it was determined that it would be necessary at least four to six years of evaluation for selection of plants, with accuracy of $80 \%$.
\end{abstract}

Index terms: Acca sellowiana, heritability, environmental factor, genetic variation.

\section{Introdução}

No melhoramento de plantas, quanto maior o número de progênies testadas, maior será a chance de captar associações alélicas desejáveis. Contudo, o número de progênies avaliadas em espécies perenes é bem menor do que em espécies anuais, uma vez

(1) Aceito para publicação em 9 de abril de 2002 .

Parte da dissertação de mestrado apresentada pelo primeiro autor à Universidade Federal de Santa Catarina, Florianópolis, SC. Apoio financeiro da Capes e do Prodetab.

(2) Universidade Federal de Santa Catarina, Dep. de Fitotecnia, Caixa Postal 476, CEP 88040-900 Florianópolis, SC. E-mail: julianadt@hotmail.com,msreis@cca.ufsc.br, mpguerra@cca.ufsc.br, nodari@mbox1.ufsc.br

${ }^{(3)}$ Empresa de Pesquisa Agropecuária e Extensão Rural de Santa Catarina, Estação Experimental, Caixa Postal 81, CEP 88600-000 São Joaquim, SC. E-mail: jeanpierre@iscc.com.br que estas ocupam maior área e apresentam longo período de juvenilidade. O sucesso e os custos desses programas estão quase diretamente relacionados ao espaço que as progênies ocupam e o tempo que ocupam nesse espaço.

A maioria das características de frutos é quantitativa, e seu desempenho flutua em decorrência dos fatores ambientais. Por isso, é importante obter informações sobre sua expressão fenotípica (Yamada et al., 1993). A avaliação dessas características em vários anos e locais pode ser necessária para propiciar uma estimativa confiável dos valores genéticos de cada indivíduo (Falconer, 1981; Fehr, 1987). Dados de apenas um ano são geralmente insuficientes quando existe interação genótipo $\mathrm{x}$ ambiente. Por isso, é comum testar essa interação para decidir se os dados devem ser coletados em mais anos, em mais de um local, ou com maior número de repetições.

A escolha do delineamento experimental também afeta a otimização. Quando se ignoram os custos, os 
delineamentos que levarem a menores variâncias das médias de genótipos são preferidos. Contudo, adicionar locais pode ser oneroso, e a adição de anos pode comprometer seriamente os programas de melhoramento (Swallow \& Wehner, 1989).

A importância do efeito de anos vem sendo demonstrada para várias culturas, perenes e anuais. Os componentes de variância ambiental e genética foram estudados em videira (Sato et al., 2000), caquizeiro (Yamada et al., 1993), noz-pecan (Thompson \& Baker, 1993), framboesa (Moore, 1997) e pepino (Swallow \& Wehner, 1989). Esses estudos vêm mostrando que aumentar o número de anos ou locais é mais eficiente para a estimação das características genéticas do que o aumento do número de progênies avaliadas. Além disso, a quantidade de recursos empregada é geralmente menor (Swallow \& Wehner, 1989; Sato et al., 1993; Yamada et al., 1993).

A determinação do número de anos necessários para que as avaliações sejam consistentes pode ser feita a partir do coeficiente de repetibilidade. Este método é obtido a partir de medidas múltiplas repetidas no tempo ou no espaço (Falconer, 1981), e utilizadas no estudo de caracteres de plantas perenes que se expressam mais de uma vez no decorrer da vida do organismo, com base na tomada de mais de uma observação fenotípica de cada indivíduo sem necessidade de recorrer a progênies a fim de medir a capacidade que os organismos têm, de repetir a expressão do caráter (Vencovsky, 1977). Este método possibilita ainda estabelecer o número mínimo de medições a serem feitas em cada indivíduo, para que se conheça seu potencial genético real, com o menor gasto de tempo e menos mão-de-obra possíveis. Este coeficiente expressa o valor máximo que a herdabilidade no sentido amplo pode atingir, já que expressa a proporção da variância fenotípica, que é atribuída às diferenças genéticas confundidas com os efeitos permanentes que estão atuando (Falconer, 1981).

Diferentes valores do coeficiente de repetibilidade vêm sendo obtidos em diferentes espécies: superiores a $60 \%$ para características de frutos em pessegueiro (Souza et al., 1998) e acerola (Gonzaga Neto et al., 1999; Lopes, 1999) e inferiores a 0,50 para produção de frutos em coqueiro (Siqueira, 1982), cajueiro-anão (Cavalcanti et al., 2000) e seringueira (Gonçalves et al., 1982; Vasconcellos et al., 1985;
Gonçalves et al., 1990). Nas características ligadas à produtividade, obtiveram-se altos valores de repetibilidade com o algodoeiro herbáceo (Farias et al., 1998) e baixos valores com a alfafa (Ferreira et al., 1999), o que indica que neste caso não houve regularidade na repetição dos caracteres no tempo.

A determinação dos efeitos ambientais e de coeficientes de repetibilidade para a goiabeira-serrana, espécie nativa do planalto meridional brasileiro, ainda não foi efetuada. Tais informações são relevantes para o programa de domesticação da espécie que apresenta uma grande variabilidade genética (Nodari et al., 1997).

Os objetivos deste trabalho foram estimar os componentes de variância ambiental, e determinar os coeficientes de repetibilidade de características dos frutos e da produtividade da goiabeira-serrana e o número de anos necessários para uma avaliação precisa.

\section{Material e Métodos}

O pomar comercial de goiabeira-serrana onde foram realizadas as avaliações está localizado no Município de São Joaquim, SC, instalado em 1985, e composto por 889 plantas. As plantas foram obtidas a partir de sementes oriundas de duas plantas-matrizes, constituindo duas famílias de meios-irmãos.

$\mathrm{O}$ efeito de anos foi estudado com base em dois grupos de dados. O primeiro grupo continha amostras de oito frutos de 26 plantas, sendo 13 de cada planta-matriz, avaliadas nos anos de 1998, 1999 e 2000 (Tabela 1). O segundo, contendo amostras de oito frutos de 54 plantas, sendo 27 de cada planta-matriz, avaliadas nos anos de 1999 e 2000 (Tabela 2). Foram avaliados: peso do fruto (PF); peso da casca (PC); rendimento da polpa, expresso em porcentagem (RP); comprimento do fruto (CF); diâmetro do fruto (DF); relação entre comprimento e diâmetro (CF/DF) e porcentagem de sólidos solúveis totais (SST).

$\mathrm{O}$ modelo genético adotado para expressar os valores fenotípicos das características foi:

$F_{i j k l}=\mu+m_{i}+p_{i j}+f_{i j k l}+a_{1}+(m a)_{i l}+(p a)_{i j l}$,

onde $\mathrm{F}_{\mathrm{ijk} k \mathrm{e}}$ o valor fenotípico do k-ésimo fruto na j-ésima progênie da i-ésima planta-matriz no l-ésimo ano; $\mu$ é a média geral; $\mathrm{m}_{\mathrm{i}}$ é o efeito aleatório da i-ésima planta-matriz; $\mathrm{p}_{\mathrm{ij}}$ é o efeito aleatório da j-ésima progênie; $\mathrm{f}_{\mathrm{ijk} \mathrm{l}}$ é o efeito aleatório do k-ésimo fruto da j-ésima progênie no l-ésimo ano; a a é o efeito aleatório do l-ésimo ano; (ma) $)_{i l}$ é a interação entre a i-ésima planta-matriz e o l-ésimo ano e $(\mathrm{pa})_{\mathrm{ijl}}$ é a interação entre a j-ésima progênie da i-ésima 
planta-matriz no l-ésimo ano. Este modelo é atribuído ao estudo de experimentos multifatoriais, em que alguns fatores são cruzados, e outros fatores, hierarquizados (Montgomery, 1991).

O modelo permite a obtenção de valores negativos dos componentes da variância, como todo método não-viesado. Os valores, quando negativos, podem ser considerados como nulos, quando o objetivo é demonstrar os componentes de variância individuais (Swallow \& Wehner, 1989).

A variância total $\left(\sigma^{2} \mathrm{~T}\right)$ foi dividida em variância de plantas-matrizes $\left(\sigma_{\mathrm{m}}^{2}\right)$, progênies dentro de plantas-matrizes $\left(\sigma_{\mathrm{p}}^{2}\right)$, frutos dentro de progênies $\left(\sigma_{\mathrm{f}}^{2}\right)$, anos $\left(\sigma_{\mathrm{a}}^{2}\right)$, a interação planta-matriz x ano $\left(\sigma^{2}\right.$ ma $)$ e a interação ano $x$ progênies dentro de planta-matriz $\left(\sigma_{\mathrm{pa}}^{2}\right)$.

No cálculo do coeficiente de repetibilidade foram avaliados de oito a dez frutos, coletados de 32 plantas tomadas ao acaso no pomar, com três repetições, referentes aos anos 1998, 1999 e 2000. As análises foram feitas com base nas médias de frutos de cada planta por ano. Foram avaliadas as características: PF, PC, RP, CF, DF, CF/DF, SST e número de frutos produzidos por planta (PRD). As estimativas dos coeficientes de repetibilidade foram obtidas a partir dos métodos: análise de variância, conforme Cruz \& Regazzi (1997) (Tabela 3); componentes principais $(\mathrm{CP})$ com base na matriz de correlações e na matriz de co-variâncias, conforme Abeywardana (1972) e Rutledge (1974); e análise estrutural (AE), com base na matriz de co-variâncias e correlações, conforme Mansour et al. (1981).

Uma vez estimada a repetibilidade (r), o número de medições $\left(\eta_{0}\right)$ necessárias para se predizer o real valor dos indivíduos com o valor do coeficiente de determinação genotípica $\left(\mathrm{R}^{2}\right)$ desejado foi obtido pela expressão:

$$
\eta_{0}=\frac{\mathrm{R}^{2}(1-\mathrm{r})}{\left(1-\mathrm{R}^{2}\right) \mathrm{r}}
$$

Tabela 1. Graus de liberdade e esperança dos quadrados médios da análise de variância de amostras de oito frutos de 13 progênies de duas plantas-matrizes, avaliadas em três anos (1998, 1999 e 2000). São Joaquim, SC.

\begin{tabular}{lrl}
\hline Fonte de variação & GL & Esperança dos quadrados médios ${ }^{(1)}$ \\
\hline Plantas-matrizes & 1 & $\sigma_{\mathrm{f}}^{2}+8 \sigma_{\mathrm{pa}}^{2}+24 \sigma_{\mathrm{p}}^{2}+104 \sigma_{\text {ma }}^{2}+312 \sigma_{\mathrm{m}}^{2}$ \\
Anos & 2 & $\sigma_{\mathrm{f}}^{2}+8 \sigma_{\mathrm{pa}}^{2}+104 \sigma_{\text {ma }}^{2}+208 \sigma_{\mathrm{a}}^{2}$ \\
Planta-matriz x anos & 2 & $\sigma_{\mathrm{f}}^{2}+8 \sigma_{\mathrm{pa}}^{2}+104 \sigma_{\mathrm{ma}}^{2}$ \\
Entre progênies dentro de planta-matriz & 24 & $\sigma_{\mathrm{f}}^{2}+8 \sigma_{\mathrm{pa}}^{2}+24 \sigma_{\mathrm{p}}^{2}$ \\
Anos x progênies dentro de planta-matriz & 48 & $\sigma_{\mathrm{f}}^{2}+8 \sigma_{\mathrm{pa}}^{2}$ \\
Entre frutos dentro de progênies & 536 & $\sigma_{\mathrm{f}}^{2}$ \\
\hline
\end{tabular}

${ }^{(1)} \sigma_{\mathrm{f}}^{2}$ : variância de frutos dentro de progênies; $\sigma_{\mathrm{pa}}^{2}$ : variância da interação ano x progênies dentro de plantas-matrizes; $\sigma^{2}$ : variância de progênies dentro de plantas-matrizes; $\sigma_{\text {ma: }}^{2}$ : variância da interação plantas-matrizes x anos; $\sigma_{\mathrm{m}}^{2}$ : variância de plantas-matrizes; $\sigma_{\mathrm{a}}^{2}$ : variância entre anos.

Tabela 2. Graus de liberdade e esperança dos quadrados médios da análise de variância de amostras de oito frutos de 27 progênies de duas plantas-matrizes avaliadas em dois anos (1999 e 2000). São Joaquim, SC.

\begin{tabular}{lrl}
\hline Fonte de variação & GL & Esperança dos quadrados médios ${ }^{(1)}$ \\
\hline Plantas-matrizes & 1 & $\sigma_{\mathrm{f}}^{2}+8 \sigma_{\mathrm{pa}}^{2}+16 \sigma_{\mathrm{p}}^{2}+216 \sigma_{\mathrm{ma}}^{2}+432 \sigma_{\mathrm{m}}^{2}$ \\
Anos & 1 & $\sigma_{\mathrm{f}}^{2}+8 \sigma_{\mathrm{pa}}^{2}+216 \sigma_{\text {ma }}^{2}+432 \sigma_{\mathrm{a}}^{2}$ \\
Planta-matriz x anos & 1 & $\sigma_{\mathrm{f}}^{2}+8 \sigma_{\mathrm{pa}}^{2}+216 \sigma_{\mathrm{ma}}^{2}$ \\
Entre progênies dentro de planta-matriz & 52 & $\sigma_{\mathrm{f}}^{2}+8 \sigma_{\mathrm{pa}}^{2}+16 \sigma_{\mathrm{p}}^{2}$ \\
Anos x progênies dentro de planta-matriz & 52 & $\sigma_{\mathrm{f}}^{2}+8 \sigma_{\mathrm{pa}}^{2}$ \\
Entre frutos dentro de progênies & 756 & $\sigma_{\mathrm{f}}^{2}$ \\
\hline
\end{tabular}

${ }^{(1)} \sigma_{\mathrm{f}}^{2}$ : variância de frutos dentro de progênies; $\sigma_{\mathrm{pa}}^{2}$ : variância da interação ano x progênies dentro de plantas-matrizes; $\sigma_{\mathrm{p}}^{2}$ : variância de progênies dentro de plantas-matrizes; $\sigma_{\mathrm{ma}}^{2}$ : variância da interação plantas-matrizes x anos; $\sigma_{\mathrm{m}}^{2}$ : variância de plantas-matrizes; $\sigma_{\mathrm{a}}^{2}$ : variância entre anos.

Tabela 3. Esquema da análise de variância do modelo com dois fatores de variação.

\begin{tabular}{lccc}
\hline Fonte de variação & GL & Quadrados médios & Esperança (QM) \\
\hline Indivíduos (I) & $\mathrm{p}-1$ & QMI & $\sigma^{2}+\eta \sigma^{2}{ }_{i}$ \\
Anos de produção (A) & $\mathrm{a}-1$ & QMA & - \\
Resíduo (R) & $(\mathrm{p}-1)(\mathrm{a}-1)$ & QMR & $\sigma^{2}$ \\
\hline
\end{tabular}


O coeficiente de determinação genotípica $\left(\mathrm{R}^{2}\right)$, que representa a certeza da predição do valor real dos indivíduos selecionados, com base em $\eta$ medições, foi obtido pela expressão:

$$
\mathrm{R}^{2}=\frac{\eta \mathrm{r}}{1+\mathrm{r}(\eta-1)}
$$

\section{Resultados e Discussão}

\section{Efeito de anos}

As análises de variância revelaram que, com exceção de SST, o efeito de anos foi significativo $(\mathrm{P}<0,01)$ para todas as características avaliadas nos dois grupos de dados (Tabela 4). A diferença entre progênies não foi significativa em relação a nenhuma das características quando foram avaliadas 26 plantas, mas foi significativa com relação a PC, DF, $\mathrm{PF}$ e CF/DF no grupo de 54 plantas, o que mostra a importância da avaliação de maior número de progênies (embora $\sigma_{\mathrm{m}}^{2}$ não tenha representado mais de $10 \%$ da variância total) na identificação de plantasmatrizes superiores (Tabela 5). Contudo, não foi importante em elevar o efeito de progênies dentro de planta-matriz.

A importância do efeito de anos foi demonstrada nos dois grupos de dados. Na análise de 26 plantas em três anos, a variância de anos contribuiu com a maior parte da variância total em três das características avaliadas (CF, DF e RP). Quanto ao grupo de 54 plantas avaliadas em dois anos, a variância de anos foi maior do que no grupo de 26 plantas em relação a todas as características, com exceção de RP. Esses valores revelaram a importância de este estudo ter sido realizado em três anos.

A importância do tamanho da amostra ficou refletida no efeito de frutos dentro de progênies, na variância total. A avaliação de um maior número de progênies por planta-matriz também deve influenciar a diminuição desse valor (Tabela 5). Amostras maiores tenderiam a diminuir os valores de variância obtidos e demandam geralmente menos recursos do que a manutenção de um maior número de plantas ou mais anos de avaliação (Tabela 5). O efeito de frutos em caquizeiro foi de $27 \%$ no tocante a peso, e $37,5 \%$ quanto a sólidos solúveis totais, demonstrando também para esta espécie a importância do tamanho da amostra de frutos (Yamada et al., 1993).

Com relação a todas as características, a soma das variâncias de ordem ambiental $\left(\sigma_{\mathrm{a}}^{2}, \sigma^{2}{ }_{\mathrm{ma}}, \sigma_{\mathrm{pa}}^{2} \mathrm{e}\right.$ $\sigma_{\mathrm{f}}^{2}$ ) representou mais de $80 \%$. Exceção ocorreu com relação a $\mathrm{CF} / \mathrm{DF}$, que foi a característica que menos sofreu influência do ambiente, o que sugere que, embora $\mathrm{CF}$ e DF apresentem grande variação ao longo dos anos, suas variações devem ocorrer de maneira

Tabela 4. Resumo da análise de variância das características peso do fruto (PF), peso da casca (PC), rendimento da polpa (RP), comprimento (CF), diâmetro (DF), relação CF/DF e sólidos solúveis totais (SST), avaliados em 26 progênies de duas plantas-matrizes em três anos e em 54 progênies de duas plantas-matrizes em dois anos de avaliação. São Joaquim, SC.

\begin{tabular}{|c|c|c|c|c|c|c|c|c|}
\hline \multirow[t]{2}{*}{ Fonte de variação } & \multirow[t]{2}{*}{ GL } & \multicolumn{7}{|c|}{ Quadrados médios } \\
\hline & & $\mathrm{PF}$ & $\mathrm{PC}$ & RP & $\mathrm{CF}$ & DF & $\mathrm{CF} / \mathrm{DF}$ & SST \\
\hline & \multicolumn{8}{|c|}{ Vinte e seis progênies } \\
\hline Plantas-matrizes & 1 & $5.723,6^{\mathrm{ns}}$ & $1.479,4^{\mathrm{ns}}$ & $425,8^{\text {ns }}$ & $2,30^{\mathrm{ns}}$ & $5,60^{\mathrm{ns}}$ & $1,10^{\mathrm{ns}}$ & $22,10^{\mathrm{ns}}$ \\
\hline Anos & 2 & $19.507,6 * *$ & $24.280,0 * *$ & $6.905,4 * *$ & $126,40 * *$ & $43,30 * *$ & $0,66^{* *}$ & $50,40^{\mathrm{ns}}$ \\
\hline Plantas-matrizes $\mathrm{x}$ anos & 2 & $1.690,1^{\text {ns }}$ & $1.180,0^{\text {ns }}$ & $160,8^{\text {ns }}$ & $4,70^{\mathrm{ns}}$ & $0,40^{\mathrm{ns}}$ & $0,10^{\mathrm{ns}}$ & $19,70^{*}$ \\
\hline Entre progênies dentro de plantas-matrizes & 24 & $3.242,3^{* *}$ & $2.322,2 * *$ & $510,0^{* *}$ & $4,10 * *$ & $2,90 * *$ & $0,38 * *$ & $11,40 *$ \\
\hline Anos $x$ progênies dentro de plantas-matrizes & 48 & $1.367,5^{* *}$ & $825,7 * *$ & $124,3^{* *}$ & $1,90 * *$ & $0,70 * *$ & $0,06^{* *}$ & $6,40^{* *}$ \\
\hline \multirow[t]{2}{*}{ Entre frutos dentro de progênies } & 536 & 305,9 & 180,9 & 22,8 & 0,30 & 0,20 & 0,01 & 1,10 \\
\hline & \multicolumn{8}{|c|}{ Cinquenta e quatro progênies } \\
\hline Plantas-matrizes & 1 & $19.711,0^{*}$ & $14.087,0 * *$ & $494,0^{\text {ns }}$ & $0,82^{\mathrm{ns}}$ & $28,40 * *$ & $2,90^{*}$ & $0,06^{\mathrm{ns}}$ \\
\hline Anos & 1 & $87.084,0 * *$ & $74.279,0 * *$ & $5.535,0^{* *}$ & $643,00 * *$ & $171,90 * *$ & $4,30^{* *}$ & $30,30^{\text {ns }}$ \\
\hline Plantas-matrizes $\mathrm{x}$ anos & 1 & $935,0^{\mathrm{ns}}$ & $537,0^{\text {ns }}$ & $47,6^{\mathrm{ns}}$ & $0,02^{\mathrm{ns}}$ & $0,88^{\text {ns }}$ & $0,03^{\mathrm{ns}}$ & $36,90^{\mathrm{ns}}$ \\
\hline Entre progênies dentro de plantas-matrizes & 52 & $3.978,0^{* *}$ & $2.648,0^{* *}$ & $380,0 * *$ & $7,22 * *$ & $2,11 * *$ & $0,34 * *$ & $9,00 * *$ \\
\hline Anos x progênies dentro de plantas-matrizes & 52 & $2.471,0 * *$ & $1.702,0 * *$ & $103,0 * *$ & $2,32 * *$ & $1,00 * *$ & $0,02 * *$ & $7,20 * *$ \\
\hline Entre frutos dentro de progênies & 756 & 339,0 & 211,0 & 23,7 & 0,35 & 0,15 & 0,01 & 0,90 \\
\hline
\end{tabular}

${ }^{n s}$ ão-significativo. * e **Significativo a $5 \%$ e a $1 \%$ de probabilidade, respectivamente. 
semelhante, não afetando de maneira muito pronunciada a razão entre ambas (CF/DF).

A influência de anos sobre SST pode ser bastante divergente entre as espécies. No maracujá-roxo, a temperatura pode agir elevando os conteúdos de SST em regiões mais quentes (Nascimento et al., 1994). Além disso, variações de 1 a $2 \%$ entre anos são consideradas comuns em caquizeiros no Japão, devido à oscilação da temperatura e da umidade (Yamada et al., 1995). Divergências ocorrem até mesmo dentro da mesma espécie. Nessa cultura sabe-se que os valores de SST são muito influenciados pelo ambiente (Yamada \& Kurihara, 1984; Yamada et al., 1993), enquanto Yamada et al. (1995) não comprovaram a importância da variância ambiental quanto a esta característica. Quanto à videira, a variância de anos foi responsável por $2 \%$ da variância total para esta característica (Sato et al., 1993).

Como observado em outras espécies frutíferas, a influência do ambiente (efeito de anos e efeito da variação dentro de plantas) sobre as características avaliadas foi grande (Tabela 5). Esses efeitos devem ter sido ainda de maior importância, pelo fato de não ter havido delineamento experimental, o qual teria permitido um controle mais rigoroso do ambiente.
Conforme constatado em videira (Sato et al., 2000) e caquizeiro (Yamada et al., 1993), o número total de progênies acomodadas no campo e avaliadas em determinado período diminuem mais pela adição de repetições do que pela adição de anos de avaliação, a menos que as plantas possam ser avaliadas já no primeiro ano. Portanto, pode-se sugerir que a avaliação da goiabeira-serrana em mais anos deva ser preferida à adição de maior número de progênies avaliadas. Essa suposição é reforçada se for considerada a magnitude das variâncias de anos obtidas. Além disso, um maior controle ambiental a partir do manejo adequado da cultura deverá diminuir a magnitude dessa variância. Amostras maiores também demonstraram ser importantes na diminuição da variância ambiental, e são, na maioria dos casos, menos onerosas.

\section{Coeficiente de repetibilidade}

O estudo para determinar o coeficiente de repetibilidade com o uso de 32 plantas em três anos mostrou haver diferenças significativas entre genótipos quanto às características, com exceção de DF, CF/DF e SST (Tabela 6). As estimativas dos coeficientes de repetibilidade obtidas a partir dos dife-

Tabela 5. Estimativas dos componentes de variância (CV) e porcentagem relativa ao total (valores entre parênteses) obtidas a partir da análise de variância para características de fruto de goiabeira-serrana das características peso do fruto (PF), peso da casca (PC), rendimento da polpa (RP), comprimento (CF), diâmetro (DF), relação CF/DF e sólidos solúveis totais (SST), avaliados em 26 progênies de duas plantas-matrizes em três anos e em 54 progênies de duas plantas-matrizes em dois anos de avaliação. São Joaquim, SC.

\begin{tabular}{|c|c|c|c|c|c|c|c|}
\hline $\mathrm{CV}^{(1)}$ & $\mathrm{PF}$ & $\mathrm{PC}$ & $\mathrm{RP}$ & $\mathrm{CF}$ & DF & $\mathrm{CF} / \mathrm{DF}$ & SST \\
\hline \multicolumn{8}{|c|}{ Vinte e seis progênies } \\
\hline$\sigma_{m}^{2}$ & $6,9(1,1)$ & $0,0(0)$ & $0,00(0)$ & $0,00(0)$ & $0,01(1,7)$ & $0,0020(5,6)$ & $0,00(0)$ \\
\hline$\sigma_{\mathrm{a}}^{2}$ & $85,7(14)$ & $111,6(25,3)$ & $32,40(38,4)$ & $0,58(46,7)$ & $0,20(38,7)$ & $0,0300(7,2)$ & $0,15(6,5)$ \\
\hline$\sigma_{\text {ma }}^{2}$ & $3,1(0,5)$ & $3,4(0,8)$ & $0,35(0,5)$ & $0,03(2,1)$ & $0,00(0)$ & $0,0004(0,8)$ & $0,13(5,7)$ \\
\hline$\sigma_{p}^{2}$ & $78,1(12,7)$ & $62,3(14,2)$ & $16,10(19,1)$ & $0,09(7)$ & $0,09(17,7)$ & $0,0100(35,9)$ & $0,21(9,4)$ \\
\hline$\sigma_{p a}^{2}$ & $132,7(21,7)$ & $80,6(18,4)$ & $12,70(15)$ & $0,20(16)$ & $0,06(12,5)$ & $0,0060(15,4)$ & $0,66(29,3)$ \\
\hline$\sigma_{f}^{2}$ & $306,0(50)$ & $181,0(41,3)$ & $22,80(27)$ & $0,35(28,2)$ & $0,15(29,4)$ & $0,0100(35,1)$ & $1,10(49,1)$ \\
\hline$\sigma_{T}^{2}$ & $612,4(100)$ & $438,4(100)$ & $84,33(100)$ & $1,25(100)$ & $0,53(100)$ & $0,3760(100)$ & $2,23(100)$ \\
\hline \multicolumn{8}{|c|}{ Cinquenta e quatro progênies } \\
\hline$\sigma_{\mathrm{m}}^{2}$ & $40,0(4,2)$ & $29,2(4,4)$ & $0,39(0,6)$ & $0,00(0)$ & $0,06(7,7)$ & $0,0060(12)$ & $0,00(0)$ \\
\hline$\sigma_{\mathrm{a}}^{2}$ & $199,4(21,2)$ & $170,7(26)$ & $12,70(19,8)$ & $1,50(62,1)$ & $0,39(50,3)$ & $0,0100(20,3)$ & $0,00(0)$ \\
\hline$\sigma_{\mathrm{ma}}^{2}$ & $0,0(0)$ & $0,0(0)$ & $0,00(0)$ & $0,00(0)$ & $0,00(0)$ & $0,0000(0)$ & $0,14(7,0)$ \\
\hline$\sigma_{p}^{2}$ & $94,2(10)$ & $59,1(9)$ & $17,30(27,1)$ & $0,31(12,8)$ & $0,07(8,8)$ & $0,0200(41,1)$ & $0,11(5,9)$ \\
\hline$\sigma_{p a}^{2}$ & $266,5(28,4)$ & $186,3(28,4)$ & $9,90(15,5)$ & $0,25(10,3)$ & $0,10(13,4)$ & $0,0020(3,3)$ & $0,79(40,5)$ \\
\hline$\sigma_{f}^{2 a}$ & $339,6(36,2)$ & $211,6(32,2)$ & $23,70(37)$ & $0,35(14,8)$ & $0,15(19,7)$ & $0,0100(23,3)$ & $0,91(46,6)$ \\
\hline$\sigma_{\mathrm{T}}^{2}$ & $939,7(100)$ & $656,9(100)$ & $64,09(100)$ & $2,41(100)$ & $0,77(100)$ & $0,0500(100)$ & $1,95(100)$ \\
\hline
\end{tabular}


rentes métodos, com relação a cada característica, foram divergentes (Tabela 7). Conforme Mansour et al. (1981), isto é esperado quando os coeficientes de repetibilidade forem pequenos. $\mathrm{O}$ valor do coeficiente inferior a 0,4 representa, de fato, dificuldades em identificar os melhores genótipos a partir da análise das médias fenotípicas obtidas, o que corrobora as altas variâncias devidas ao efeito de anos, obtidas na análise do efeito de anos. A predição do valor real, expressa pelo coeficiente de determinação, foi inferior a $70 \%$ para a maioria das características, o que indica que a superioridade ou inferioridade do comportamento dos genótipos pode não ser mantida.

Os valores mais elevados de repetibilidade do RP indicam que a variância ambiental desta característica entre anos foi menor. Esses valores divergem daqueles obtidos para a análise de efeito de anos, onde o RP foi uma das características com maior variância de anos para o grupo de plantas avaliado em três anos.
Os valores de repetibilidade do PF (entre 0,39 e 0,41 ) podem ser considerados baixos, em comparação com o encontrado nas outras espécies, como caquizeiro $(0,77)$ (Yamada \& Kurihara, 1984), cupuaçuzeiro $(0,76)$ (Costa et al., 1997), e acerola $(0,65)$ (Gonzaga Neto et al., 1999; Lopes, 1999). Esses valores podem estar sendo influenciados também pela falta de manejo adequado, o qual pode influenciar as características dos frutos nos diferentes anos. O raleio, por exemplo, é uma prática que tende a aumentar e homogeneizar o tamanho dos frutos (Manica et al., 2000). Em outras espécies, a idade das plantas também pode influenciar o peso de frutos, como em citros, onde ele se tornou menor ao longo dos cinco ciclos avaliados (Ogata \& Mendonça, 2000).

Constata-se, então, a necessidade de quatro a seis medições para obter dados com precisão de $80 \%$ das características, com exceção do CF e SST, para as quais seriam necessárias 10 e 23 medições, respecti-

Tabela 6. Resumo da análise de variância dos caracteres peso do fruto (PF), peso da casca (PC), rendimento da polpa (RP), comprimento (CF), diâmetro (DF), relação CF/DF, sólidos solúveis totais (SST) e produtividade (PRD), em amostras de 10 frutos avaliados de 32 plantas, nos anos de 1998, 1999 e 2000. São Joaquim, SC.

\begin{tabular}{lrcccccccc}
\hline Fonte de & GL & \multicolumn{10}{c}{ Quadrados médios } \\
\cline { 2 - 10 } variaça & & PF & PC & RP & CF & DF & CF/DF & SST & PRD $^{(1)}$ \\
\hline Anos & 2 & $2.100,0$ & $2.739,1$ & 972,2 & 18,6 & 6,1 & 9,2 & 10,3 & $73.602,2$ \\
Indivíduos & 31 & $426,3^{* *}$ & $279,9^{* *}$ & $66,0^{* *}$ & $0,5^{* *}$ & $0,4^{\text {ns }}$ & $5,0^{\text {ns }}$ & $1,3^{\text {ns }}$ & $4.861,6^{* *}$ \\
Resíduo & 62 & 143,6 & 95,9 & 15,7 & 0,2 & 7,3 & 9,2 & 0,9 & $1.618,7$ \\
\hline Média & 72,5 & 52,3 & 28,5 & 5,6 & 4,5 & 1,2 & 10,5 & 70,0 \\
CV $(\%)$ & & 16,5 & 18,7 & 13,9 & 8,9 & 6,0 & 7,7 & 9,2 & 37,5 \\
\hline
\end{tabular}

${ }^{(1)}$ Graus de liberdade de indivíduos igual a 99 e de resíduo igual a 198, correspondentes à avaliação de 100 plantas. nsNão-significativo. **Significativo a $1 \%$ de probabilidade.

Tabela 7. Estimativas do coeficiente de repetibilidade dos caracteres peso do fruto (PF), peso da casca (PC), rendimento da polpa (RP), comprimento $(\mathrm{CF})$, diâmetro $(\mathrm{DF})$, relação $\mathrm{CF} / \mathrm{DF}$, sólidos solúveis totais (SST) e produtividade (PRD), com base em amostras de 10 frutos avaliados de 32 plantas, nos anos de 1998, 1999 e 2000. São Joaquim, SC ${ }^{(1)}$.

\begin{tabular}{|c|c|c|c|c|c|}
\hline \multirow[t]{2}{*}{ Caráter } & \multirow[t]{2}{*}{ ANOVA } & \multicolumn{2}{|c|}{ Componentes principais } & \multicolumn{2}{|c|}{ Análise estrutural } \\
\hline & & Co-variância & Correlação & Co-variância & Correlação \\
\hline $\mathrm{PF}$ & $0,396(66,31)$ & $0,410(67,56)$ & $0,396(66,32)$ & $0,395(66,22)$ & $0,396(66,31)$ \\
\hline $\mathrm{PC}$ & $0,390(65,73)$ & $0,418(68,32)$ & $0,394(66,13)$ & $0,394(66,07)$ & $0,390(65,73)$ \\
\hline $\mathrm{RP}$ & $0,517(76,28)$ & $0,595(81,55)$ & $0,535(77,55)$ & $0,534(77,48)$ & $0,517(76,28)$ \\
\hline $\mathrm{CF}$ & $0,277(53,56)$ & $0,284(54,30)$ & $0,286(54,63)$ & $0,283(54,28)$ & $0,278(53,56)$ \\
\hline DF & $0,595(81,52)$ & $0,634(83,85)$ & $0,602(81,91)$ & $0,600(81,82)$ & $0,595(81,52)$ \\
\hline $\mathrm{CF} / \mathrm{DF}$ & $0,600(81,8)$ & $0,650(85,0)$ & $0,630(83,6)$ & $0,620(83,4)$ & $0,600(81,8)$ \\
\hline SST & $0,120(29,12)$ & $0,162(36,72)$ & $0,166(37,37)$ & $0,149(34,45)$ & $0,120(29,12)$ \\
\hline PRD & $0,400(66,70)$ & $0,482(73,66)$ & $0,402(66,86)$ & $0,388(65,60)$ & $0,400(66,70)$ \\
\hline
\end{tabular}

(1)Valores entre parênteses referem-se ao coeficiente de determinação (\%) associado ao coeficiente de repetibilidade. 
vamente (Tabela 8). Contudo, os valores obtidos para DF e SST devem ser tomados com cautela, uma vez que a análise de variância não detectou diferenças significativas entre genótipos, e este método leva em consideração a variância entre genótipos e a variância dentro de genótipos. Os baixos valores encontrados para DF e CF/DF e os altos valores para SST devem estar sendo influenciados pela reduzida variação entre plantas.

Valores considerados baixos de repetibilidade $(<0,60)$ e de coeficiente de determinação reduzidos não são exclusivos da goiabeira-serrana, pois foram encontrados no que se refere a porcentagem de polpa e de fibra em cupuaçu, quando não houve diferença estatística entre os indivíduos na análise de variância (Costa et al., 1997). Esse fato pode ser comprovado pela diferença entre os coeficientes calculados para CF e DF, características que variam de maneira similar na população avaliada como um todo (Tabela 7). Porém, diferença bastante grande entre os coeficientes dessas características ocorreu também para o cupuaçuzeiro, no qual o comprimento e o diâmetro apresentaram $r=0,81$ e r $=0,50$, respectivamente (Costa et al., 1997).

$\mathrm{O}$ fato de o coeficiente baseado na análise dos componentes principais ter apresentado o maior valor para a característica produtividade pode sugerir a existência de alternância de produção da espécie, uma vez que este método de determinação é mais utilizado quando os genótipos avaliados apresentam comportamento cíclico em relação ao caráter ava-

Tabela 8. Número de medições associado aos coeficientes de determinação $\left(\mathrm{R}^{2}\right)$ de 0,80 e 0,90 , com base na maior repetibilidade estimada para os caracteres peso do fruto $(\mathrm{PF})$, peso da casca $(\mathrm{PC})$, rendimento da polpa (RP), comprimento $(\mathrm{CF})$, diâmetro $(\mathrm{DF})$, relação $\mathrm{CF} / \mathrm{DF}$, sólidos solúveis totais (SST) e produtividade (PRD), em amostras de 10 frutos avaliados de 32 plantas, nos anos de 1998 , 1999 e 2000 . São Joaquim, SC.

\begin{tabular}{lccc}
\hline Caracteres & Repetibilidade & $\mathrm{R}^{2}=0,80$ & $\mathrm{R}^{2}=0,90$ \\
\hline PF & 0,409 & 6 & 14 \\
PC & 0,418 & 6 & 14 \\
RP & 0,596 & 4 & 8 \\
CF & 0,286 & 10 & 21 \\
DF & 0,633 & 3 & 6 \\
CF/DF & 0,650 & 3 & 5 \\
SST & 0,166 & 23 & $>45$ \\
PRD & 0,482 & 5 & 10 \\
\hline
\end{tabular}

liado (Abeywardana, 1972). Em relação ao cajueiroanão precoce, com apenas duas medições, obter-seia resultado com precisão de $90 \%$ nesta característica (Cavalcanti et al., 2000). O valor do coeficiente no coqueiro-comum foi de 0,45 (Siqueira, 1982) e de 0,61 para a acerola (Gonzaga Neto et al., 1999), o que demonstra a existência de divergência entre as espécies.

As análises decorrentes dos três anos de avaliação em goiabeira-serrana seriam insuficientes para a seleção de indivíduos com confiabilidade de 80 e $90 \%$. Porém, tomando a repetibilidade como limite da herdabilidade, os valores obtidos podem ser considerados razoáveis em termos de progresso genético.

De maneira geral, os resultados das duas análises foram coerentes, com exceção de RP, que apresentou variância de anos bastante acentuada no grupo de 26 plantas, no estudo de efeito de anos. A comparação entre as duas análises sugere mais uma vez que os valores de repetibilidade obtidos para DF devem ser tomados com cautela, uma vez que sua variação na análise do efeito de anos foi bastante grande, representando mais de $50 \%$ para o grupo de 54 plantas, variando de maneira similar ao comprimento. O mesmo ocorreu com relação ao valor da repetibilidade de SST e CF/DF.

\section{Conclusões}

1. Os efeitos ambientais afetam significativamente as características de frutos avaliados, e o efeito de anos é o de maior magnitude.

2. Como a variância de anos no grupo de plantas avaliado por três anos foi menor do que no grupo avaliado por dois anos, é recomendável que a avaliação seja feita por períodos mais prolongados.

3 . Os valores de repetibilidades, embora pequenos, corroboram a necessidade do estudo do efeito de anos, e demonstram a necessidade de avaliação das características estudadas em, no mínimo, quatro anos.

4. As divergências encontradas com relação a SST e DF entre as duas análises reforçam o grande efeito da variação entre plantas no cálculo do coeficiente de repetibilidade.

\section{Agradecimentos}

À Capes, pela concessão de bolsa à primeira autora; ao senhor Shu Otani, proprietário do pomar, por ter permitido a realização deste trabalho. 


\section{Referências}

ABEYWARDANA, V. An application of component analysis in genetics. Journal of Genetics, Bangalore, v. 61, p. 27-51, 1972.

CAVALCANTI, J. R. V.; PAIVA, J. R.; BARROS, L. M.; CRISÓSTOMO, J. R.; CORREA, M. P. F. Repetibilidade de caracteres de produção e porte da planta em clones de cajueiro-anão precoce. Pesquisa Agropecuária Brasileira, Brasília, v. 35, n. 4, p. 773-777, abr. 2000.

COSTA, J. G.; LEDO, A. S.; OLIVEIRA, M. N. Estimativas de repetibilidade de características de frutos de cupuaçuzeiro no Estado do Acre. Revista Brasileira de Fruticultura, Cruz das Almas, v. 19, n. 3, p. 313-318, 1997.

CRUZ, C. D.; REGAZZI, A. J. Modelos biométricos aplicados ao melhoramento genético. 2. ed. Viçosa, MG: UFV, 1997. 390 p.

FALCONER, D. S. Introduction to quantitative genetics. 2. ed. London: Longman, 1981. 340 p.

FARIAS, F. J. C.; RAMALHO, M. A. P.; CARVALHO, L. P.; MOREIRA, J. A. N.; COSTA, J. N. Repetibilidade dos parâmetros de estabilidade na cultura do algodoeiro herbáceo. Pesquisa Agropecuária Brasileira, Brasília, v. 33, n. 4, p. 457-461, abr. 1998.

FEHR, W. R. Principles of cultivar development, theory and technique. New York: Macmillan, 1987. v. 1.

FERREIRA, R. P.; BOTREL, M. A.; PEREIRA, A. V.; CRUZ, C. D. Avaliação de cultivares de alfafa e estimativas de repetibilidade de caracteres forrageiros. Pesquisa Agropecuária Brasileira, Brasília, v. 34, n. 6, p. 995 1002, jun. 1999.

GONÇALVES, P. S.; CARDOSO, M.; SÁES, L. A. Estimativas de repetibilidade na seleção de árvores adultas de seringueira. Pesquisa Agropecuária Brasileira, Brasília, v. 25, n. 7, p. 1031-1038, jul. 1990.

GONÇALVES, P. S.; ROSSETTI, A. G.; PAIVA, J. R. Coeficiente de repetibilidade e eficiência do miniteste de produção na seleção de plantas de seringueira. Pesquisa Agropecuária Brasileira, Brasília, v. 17, n. 2, p. 233-237, fev. 1982.

GONZAGA NETO, L.; MATTUZ, B.; SANTOS, C. A. F. Caracterização agronômica de clones de aceroleira (Malpighia spp.) na região do submédio São Francisco. Revista Brasileira de Fruticultura, Jaboticabal, v. 21, n. 2, p. 110-115, 1999.
LOPES, R. Polimorfismo, sistema de acasalamento, polinizações, repetibilidade de características do fruto e avaliação de genótipos de acerola. 1999. $146 \mathrm{f}$. Tese (Mestrado) - Universidade Federal de Viçosa, Viçosa.

MANICA, I.; ICUMA, I. M.; JUNQUEIRA, N. T. V.; SALVADOR, J. O.; MOREIRA, A.; MALAVOLTA, E. Fruticultura tropical 6: goiaba. Porto Alegre: Cinco Continentes, 2000. 374 p.

MANSOUR, H.; NORDHEIM, E. V.; RUTLEDGE, J. J. Estimations of repeatability. Theoretical and Applied Genetics, Berlin, v. 60, p. 151-156, 1981.

MONTGOMERY, D. C. Design and analysis of experiments. 3. ed. New York: Wiley, 1991. 461 p.

MOORE, P. P. Year-to-year consistency of harvest data in raspberry breeding plots. Journal of the American Society for Horticultural Science, Alexandria, v. 122, n. 2, p. 211-214, 1997.

NASCIMENTO FILHO, F. J. do; GARCIA, T. B.; CRUZ, C. D. Estimativa de parâmetros genéticos em clones de guaranazeiro. Pesquisa Agropecuária Brasileira, Brasília, v. 29, n. 1, p. 91-96, jan. 1994.

NODARI, R. O.; DUCROQUET, J. P. H. J.; GUERRA, M. P.; MELER, K. Genetic variability of Feijoa sellowiana germoplasm. Acta Horticulturae, Leuven, n. 452, p. 4146, 1997.

OGATA, T.; MENDONÇA, J. L. Avaliação de clones de laranja pêra, em Anápolis. Revista Brasileira de Fruticultura, Jaboticabal, v. 22, n. 1, p. 24-26, 2000.

RUTLEDGE, J. J. A scaling which removes bias of Abeywardana's estimator of repeatability. Journal of Genetics, Bangalore, v. 61, p. 247-250, 1974.

SATO, A.; YAMADA, M.; IWANAMI, H.; HIRAKAWA, N. Optimal spatial and temporal measurement repetition for reducing environmental variation of berry traits in grape breeding. Scientia Horticulturae, Amsterdam, v. 85, p. 75-83, 2000.

SATO, A.; YAMADA, M.; YAMANE, H.; HIRAKAWA, N. Repeatability and environmental variation of fruit traits in seedling population of grapes. Bulletin of the Fruit Tree Research Station, Hiroshima, n. 24, p. 1-11, 1993.

SIQUEIRA, E. R. Coeficiente de repetibilidade da produção de frutos de coqueiro comum. Pesquisa Agropecuária Brasileira, Brasília, v. 17, n. 4, p. 573-574, abr. 1982. 
SOUZA, V. A. B.; BYRNE, D.; TAYLOR, J. F. Heritability, genetic and phenotypic correlations, and predicted selection response of quantitative traits in peach II: an analysis of several fruit traits. Journal of the American Society for Horticultural Science, Alexandria, v. 123, n. 4, p. 604-611, 1998.

SWALLOW, W. H.; WEHNER, T. Optimum allocation of plots to years, seasons, locations, and replications, and its application to once-over-harvest cucumber trials. Euphytica, Dordrecht, v. 43, p. 59-68, 1989.

THOMPSON, T. E.; BAKER, J. F. Heritability and phenotypic correlations of six pecan nut characteristics. Journal of the American Society for Horticultural Science, Alexandria, v. 118, n. 3, p. 415-418, 1993.

VASCONCELLOS, M. E. C.; GONÇALVES, P. S.; PAIVA, J. R.; VALOIS, A. C. C. Métodos de estimação do coeficiente de repetibilidade no melhoramento da serin- gueira. Pesquisa Agropecuária Brasileira, Brasília, v. 20, n. 4, p. 433-437, abr. 1985.

VENCOVSKY, R. Repetibilidade. In: PRINCÍPIOS de genética quantitativa. Piracicaba: Esalq, 1977. p. 47-52.

YAMADA, M.; KURIHARA, A. Repeatability of fruit characters in pollination constant non-astringent seedling population of Japanese persimmon. Bulletin of the Fruit Tree Research Station, Hiroshima, v. 5, p. 1-8, 1984.

YAMADA, M.; WANG, R.; YAMANE, H.; SATO, A.; HIRAKAWA, N. Comparisons in the variations in fruit maturing time, fruit weight, and soluble solids content of oriental persimmon cultivars of Chinese and Japanese origin. Journal of the Japanese Society of Horticultural Science, v. 64, n. 2, p. 227-233, 1995.

YAMADA, M.; YAMANE, H.; YOSHINAGA, K.; UKAI, Y. Optimal spatial and temporal measurement repetition for selection in Japanese persimmon breeding. HortScience, Alexandria, v. 28, n. 8, p. 838-841, 1993. 